\title{
Astronomy and Astrophysics from Antarctica: a new SCAR Scientific Research Program
}

\author{
J. W. V. Storey \\ School of Physics, University of New South Wales, Sydney NSW 2052, Australia \\ email: j.storey@unsw.edu.au
}

\begin{abstract}
In July 2008 the IAU became a union member of the ICSU body SCAR - the Scientific Committee on Antarctic Research. At the same time, SCAR initiated a Planning Group to establish a Scientific Research Program in Astronomy and Astrophysics from Antarctica. Broadly stated, the objectives of Astronomy and Astrophysics from Antarctica are to coordinate astronomical activities in Antarctica in a way that ensures the best possible outcomes from international investment in Antarctic astronomy, and maximizes the opportunities for productive interaction with other disciplines.
\end{abstract}

Keywords. Antarctica, the Arctic, Site Testing

\section{What is SCAR?}

SCAR - the Scientific Committee on Antarctic Research — was established as an ICSU body in 1957 and held its first meeting in 1958. It currently has 31 Full Members (those countries with active scientific research programme in Antarctica), 4 Associate Members (those countries without an independent research programme as yet or which are planning a research programme in the future) and, with the recent addition of the IAU, 9 Union Members (those ICSU scientific unions that have an interest in Antarctic research). Like the IAU, SCAR and its divisions hold a number of meetings and conferences throughout the year. Every two years an Open Science Conference is held, similar in size and breadth to the IAU General Assemblies. More information on SCAR is available from the SCAR web site: http://www.scar.org/.

SCAR is organised into three Standing Scientific Groups. These are the:

- Standing Scientific Group on GeoSciences

- Standing Scientific Group on Life Sciences

- Standing Scientific Group on Physical Sciences

In addition, there are five Scientific Research Programmes (SRPs) whose focus is on international scientific coordination. Currently, these are:

- Antarctic Climate Evolution (ACE)

- Subglacial Antarctic Lake Environments (SALE)

- Evolution and Biodiversity in the Antarctic (EBA)

- Antarctica and the Global Climate System (AGCS)

- Interhemispheric Conjugacy Effects in Solar-Terrestrial and Aeronomy Research (ICESTAR)

At the end of 2009, ICESTAR will no longer be an SRP, making way for Astronomy and Astrophysics from Antarctica (AAA) as a new Scientific Research Program. 


\section{Astronomy and Astrophysics from Antarctica (AAA)}

AAA has set for itself the following goals. To:

(a) Coordinate site-testing experiments to ensure that results obtained from different sites are directly comparable and well understood,

(b) Build a data base of site-testing data that is accessible to all researchers,

(c) Increase the level of coordination and cooperation between astronomers, atmospheric physicists, space physicists and meteorologists,

(d) Extend existing Antarctic site-testing and feasibility studies to potential Arctic sites; for example, in Greenland and Canada,

(e) Define and prioritise current scientific goals,

$(f)$ Create a roadmap for development of major astronomical facilities in Antarctica,

(g) Stimulate international cooperation on major new astronomical facilities in Antarctica.

\section{Data Archiving}

Section III.1.c of the Antarctic Treaty (1959) states that "Scientific observations and results from Antarctica shall be exchanged and made freely available." To assist with meeting this requirement, SCAR has established the Standing Committee on Antarctic Data Management (SCADM). SCADM helps facilitate co-operation between scientists and nations with regard to scientific data, and advises on the development of the Antarctic Data Management System. AAA will work closely with SCADM to maximise the usefulness and accessibility of data collected by astronomers in Antarctica.

\section{Structure of AAA}

To achieve its goals, AAA will be structured as four themes:

- Site testing, validation and data archiving.

- Arctic site testing.

- Science goals.

- Major new facilities.

Each of these themes will be managed by a working group. Each working group has a chair and a vice-chair, and a variable number of members. All IAU members, and others, are warmly encouraged to join one or more of these working groups and to contribute to the success of the AAA program.

\section{Acknowledgements}

I thank the other members of the AAA SRP Planning Group for their assistance in putting the program together: Michael Andersen, Philip Anderson, Michael Burton, Xiangqun Cui, Nicolas Epchtein, Takashi Ichikawa, Albrecht Karle, James Lloyd, Silvia Masi \& Lifan Wang

\section{References}

The Antarctic Treaty, 1959. Available at http://www.ats.aq/ (Accessed September 2009). 